Revista de Matemática: Teoría y Aplicaciones 3(1): 61-70 (1996)

\title{
EL INCREMENTO MULTIVARIADO
}

\author{
Winston Alarcón Athens ${ }^{1}$
}

\begin{abstract}
Resumen
Desarrollamos y estudiamos una generalización a funciones multivariadas de la noción de incremento, mediante la cual pueden reproducirse en varias variables, diversas definiciones y teoremas importantes del Cálculo en una variable. En particular, es posible reproducir de manera isomorfa la reelaboración de la integral de Riemann basada en la noción de pre-primitiva, expuesta en [1]. Esto será tema de otro artículo.
\end{abstract}

\begin{abstract}
We develop and study a generalization to multivariate functions of the notion of increment, by mean of we can reproduce in several variables, many importants theorems of the Calculus in one variable. In particular, it is possible to reproduce isomorfically the reelaboration of the Riemann integral based on the notion of preprimitive, exposed in [1]. This will be the treated in a future paper.
\end{abstract}

\section{Introducción}

Seguramente al lector le ha llamado la atención que la unidad interna del Cálculo Diferencial y el Cálculo Integral — esto es, el Teorema Fundamental del Cálculo (TFC) aparezca en toda su plenitud sólo para funciones de una sola variable real. El hecho que el teorema de Stokes ([3], [4]) sea una generalización a $\mathbb{R}^{n}$ del TFC, no es suficiente para disipar esa impresión, ya que en el Teorema de Stokes se plantea una relación entre integrales y no una relación entre la integral de una derivada y la función sin derivar. Por su parte, el recurso al teorema de Fubini ([3], [4]), sólo reafirma aquella impresión.

En esta misma linea de observaciones, probablemente el lector también ha notado que tenemos teorema de Rolle ([2]) sólo en una variable y que las versiones en varias variables del teorema del Valor Medio para derivadas ([2]), no son completamente isomorfas a su versión en una variable.

El origen de estas y otras diferencias entre el Cálculo en una y en varias variables, parece radicar en la manera como se generaliza a $\mathbb{R}^{n}$ la noción de incremento de una función $f(x)$ desde $x=a$ hasta $x=b$, manteniendo la misma expresión formal

$$
\left.\Delta f(x)\right|_{a} ^{b}=f(b)-f(a),
$$

\footnotetext{
${ }^{1}$ Escuela de Matemática, Universidad de Costa Rica, 2060 San José, Costa Rica
} 
tanto si $x$ es una variable real y $a, b$ son puntos de $\mathbb{R}$, como si $x=\left(x_{1}, x_{2}, \ldots, x_{n}\right)$ es una variable $n$-dimensional y $a=\left(a_{1}, a_{2}, \ldots, a_{n}\right), b=\left(b_{1}, b_{2}, \ldots, b_{n}\right)$ son puntos de $\mathbb{R}^{n}$.

Esta noción habitual de incremento juega un rol central bastante uniforme en todo el Cálculo Diferencial, tanto en una como en varias variables. Por ejemplo, en la definición de derivada de una función de una variable real y en la definición de diferencial de una función de varias variables reales, la noción habitual de incremento juega un rol central y uniforme en implicaciones del tipo

$$
\left\|\left.\Delta x\right|_{a} ^{z}\right\|<\delta \Longrightarrow|\Delta f(x)|_{a}^{z}-\lambda\left(\left.\Delta x\right|_{a} ^{z}\right)\left|<\epsilon\left\|\left.\Delta x\right|_{a} ^{z}\right\|\right.
$$

Es probable que este uso central y uniforme de la noción habitual de incremento se origine en el hecho que el desarrollo clásico del Cálculo Diferencial en una y en varias variables estuvo motivado por problemas en los que se requiere resolver desigualdades del tipo

$$
f(y) \geq f(z)
$$

(como ocurre, por ejemplo, en los problemas de extremos), esto es, desigualdades reducibles a la forma

$$
\left.\Delta f(x)\right|_{y} ^{z} \leq 0 .
$$

En cambio, en el Cálculo Integral, el papel que juega la noción habitual de incremento sufre un brusco cambio, al pasar de una a varias variables reales. Esto se observa claramente en el distinto rol desempeñado por la noción habitual de incremento en las sumas de Riemann, según se trate de funciones de una o de varias variables: mientras en una variable, las sumas de Riemann de una función $f(x)$ hacen uso de los incrementos de la variable $x$, en varias variables aparece en su reemplazo el producto de los incrementos de las $n$ componentes reales $x_{i}$ de la variable $n$-dimensional $x=\left(x_{1}, \ldots, x_{n}\right)$.

Estas consideraciones sugieren que para obtener en varias variables, versiones isomorfas de los teoremas del Cálculo en una variable, hay que desarrollar una nueva generalización a $\mathbb{R}^{n}$ de la noción de incremento en $\mathbb{R}$, de manera que el producto de incrementos de las componentes $x_{i}$ de la variable $n$-dimensional $x=\left(x_{1}, \ldots, x_{n}\right)$ corresponda - bajo esa generalización - al incremento de la variable $n$-dimensional $x=\left(x_{1}, \ldots, x_{n}\right)$.

Uno aprende a convivir con las comentadas disimilitudes entre el Cálculo en una y en varias variables, hasta que encuentra una buena razón para encararlas. El autor ha encontrado una buena razón al tratar de generalizar a varias variables su enfoque de la integral de Riemann en una variable mediante la noción de pre-primitiva ([1]), tema que tratará en una próxima publicación.

\section{La noción de incremento multivariado}

Para generalizar a $\mathbb{R}^{n}$ la noción de incremento en $\mathbb{R}$ nos guiaremos por la discusión anterior, exigiendo que expresiones tales como $\left(b_{1}-a_{1}\right)\left(b_{2}-a_{2}\right)$, que aparecen en los términos de las sumas de Riemann de una función de dos variables, correspondan - bajo la generalización que buscamos - al incremento de la variable $x=\left(x_{1}, x_{2}\right)$ desde $a=\left(a_{1}, a_{2}\right)$ hasta $b=\left(b_{1}, b_{2}\right)$, o que expresiones como $\left(b_{1}-a_{1}\right)\left(b_{2}-a_{2}\right)\left(b_{3}-a_{3}\right)$ correspondan al 
incremento de la variable $x=\left(x_{1}, x_{2}, x_{3}\right)$ desde $a=\left(a_{1}, a_{2}, a_{3}\right)$ hasta $b=\left(b_{1}, b_{2}, b_{3}\right)$. Al desarrollar estas expresiones, obtenemos:

$$
\begin{array}{rr}
\left(b_{1}-a_{1}\right)\left(b_{2}-a_{2}\right)= & b_{1} b_{2}-b_{1} a_{2}-a_{1} b_{2}+a_{1} a_{2} \\
\left(b_{1}-a_{1}\right)\left(b_{2}-a_{2}\right)\left(b_{3}-a_{3}\right)= & b_{1} b_{2} b_{3}-b_{1} a_{2} b_{3}-a_{1} b_{2} b_{3}-b_{1} b_{2} a_{3} \\
& \quad+a_{1} a_{2} b_{3}+b_{1} a_{2} a_{3}+a_{1} b_{2} a_{3}-a_{1} a_{2} a_{3} .
\end{array}
$$

Se observa que el incremento que buscamos definir para una función $f\left(x_{1}, \ldots, x_{n}\right)$ desde el "punto inicial" $a=\left(a_{1}, \ldots, a_{n}\right)$ hasta el "punto final" $b=\left(b_{1}, \ldots, b_{n}\right)$, es una combinación lineal de los valores de $f$ en los $2^{n}$ puntos $\left(x_{1}, \ldots, x_{n}\right)$ de $\mathbb{R}^{n}$ que se obtienen al darle a cada coordenada $x_{i}$ el valor $a_{i}$ o bien el valor $b_{i}$; los coeficientes en tal combinación lineal son $(+1)$ o $(-1)$, según que el número de coordenadas del punto inicial $a$ que intervengan en el respectivo término sea par o impar. Además se observa que el "incremento de la variable $x=\left(x_{1}, \ldots, x_{n}\right)$ " es el que corresponde a la función $f\left(x_{1}, \ldots, x_{n}\right)=x_{1} \cdot \ldots \cdot x_{n}$.

Esto nos sugiere partir con la siguiente definición auxiliar:

\section{Definición A.}

- i) Dado $n=1,2,3, \ldots$ y dado $K=\left(K_{1}, \ldots, K_{n}\right) \in\{0,1\}^{n}$, definimos:

$$
|K|=\sum_{i=1}^{n} K_{i}
$$

- ii) Dados $a=\left(a_{1}, \ldots, a_{n}\right), b=\left(b_{1}, \ldots, b_{n}\right) \in \mathbb{R}^{n}$, definimos la aplicación $(a \vee b)$ de $\{0,1\}^{n}$ en $\mathbb{R}^{n}$, mediante:

$$
(a \vee b)(K)_{j}= \begin{cases}b_{j} & \text { si } K_{j}=0 \\ a_{j} & \text { si } K_{j}=1\end{cases}
$$

A continuación estamos en condiciones de formular la definición principal:

\section{Definición B.}

- i) Dada una $n$-celda $J \in \mathbb{R}^{n}$ y dada una función real $f$ definida en $J$, para cada $(a, b) \in J \times J$ definimos el incremento multivariado de $f$ desde a hasta $b$, mediante

$$
{ }^{a} \Delta^{b} f(x)=\sum_{K \in\{0,1\}^{n}}(-1)^{|K|} f((a \vee b)(K))
$$

- ii) En particular, dados $a=\left(a_{1}, \ldots, a_{n}\right), b=\left(b_{1}, \ldots, b_{n}\right) \in \mathbb{R}^{n}$, definimos el incremento multivariado de la variable $x=\left(x_{1}, \ldots, x_{n}\right)$ desde a hasta $b,{ }^{a} \Delta^{b} x$, como el incremento multivariado desde $a$ hasta $b$ de la función $f\left(x_{1}, x_{2}, \ldots, x_{n}\right)=x_{1} \cdot x_{2} \cdot \ldots \cdot x_{n}$ :

$$
{ }^{a} \Delta^{b} x={ }^{a} \Delta^{b}\left(x_{1} \cdot x_{2} \cdot \ldots \cdot x_{n}\right)
$$


- iii) Dada una $n$-celda $J=\left[a_{1}, b_{1}\right] \times \cdots \times\left[a_{n}, b_{n}\right] \subseteq \mathbb{R}^{n}$, con $a_{i} \leq b_{i}$ para todo $i=1, \ldots, n$ y dada una función real $f$ definida en $J$, definimos el incremento de $f$ en $J$ mediante

$$
\Delta_{J} f={ }^{a} \Delta^{b} f(x)
$$

donde $a=\left(a_{1}, \ldots, a_{n}\right)$ y $b=\left(b_{1}, \ldots, b_{n}\right)$.

Algunos autores (ver, por ejemplo, [2], pág. 368) utilizan la noción de "diferencia mezclada" de una función $f(x, y)$ como auxiliar técnico ad-hoc en la prueba de ciertos teoremas sobre derivadas parciales mixtas de segundo orden. Esa noción corresponde a nuestra definición de incremento multivariado de $f$ desde $(0,0)$ hasta $(h, k)$, para el caso particular $n=2$ :

$$
{ }^{(0,0)} \Delta^{(h, k)} f(x)=f(h, k)-f(h, 0)-f(0, h)+f(0,0)
$$

\section{Interpretación física del incremento multivariado}

Si $\left(x_{0}, y_{0}\right)$ es un punto del plano y si para cada par de números reales $x \geq x_{0}, y \geq y_{0}$, $\mu(x, y)$ denota una magnitud aditiva (como el área, la masa, la carga eléctrica) asociada a la 2 -celda de vértices opuestos $\left(x_{0}, y_{0}\right),(x, y)$, entonces la respectiva magnitud $\mu(J)$ de cualquier 2-celda $J \subseteq\left[x_{0}, \infty\left[\times\left[y_{0}, \infty[\right.\right.\right.$ es precisamente el incremento de $\mu$ en $J$.

Debido al teorema 3 acerca de la aditividad del incremento sobre una $n$-celda $J$ respecto de las particiones de $J$ en subceldas, esta interpretación puede generalizarse a $n$-dimensiones.

A fin de relacionar la noción de incremento multivariado con la noción habitual de incremento, daremos la siguiente definición:

Definición C. Dado $n=1,2,3, \ldots$; dados $n$ intervalos $J_{i} \subseteq \mathbb{R}(i=1, \ldots, n)$; dada una función real $f$ definida en la $n$-celda $J=J_{1} \times \cdots \times J_{n} \subseteq \mathbb{R}^{n} ;$ y dado $i=1, \ldots, n$, definimos el incremento parcial de $f$ respecto de la $i$-ésima variable, desde $a_{i} \in J_{i}$ hasta $b_{i} \in J_{i}$, mediante:

$$
\left.\Delta_{i} f(x)\right|_{a_{i}} ^{b_{i}}=f\left(x_{1}, \ldots, x_{i-1}, b_{i}, x_{i+1}, \ldots, x_{n}\right)-f\left(x_{1}, \ldots, x_{i-1}, a_{i}, x_{i+1}, \ldots, x_{n}\right) .
$$

Obsérvese que si $f$ es una función de $n$ variables, el incremento parcial ${ }^{a_{i}} \Delta_{i}^{b_{i}} f(x)$ es una función de las $n-1$ variables $\left(x_{1}, \ldots, x_{i-1}, x_{i+1}, \ldots, x_{n}\right)$.

\section{Propiedades fundamentales}

Teorema 1. (Fórmula de recurrencia). Sea $J \subseteq \mathbb{R}^{n}$ una $n$-celda, $f$ una función real definida en $J$, y $p$ una permutación de $\{1,2, \ldots, n\}$. Entonces, para cada $a=\left(a_{1}, \ldots, a_{n}\right)$, $b=\left(b_{1}, \ldots, b_{n}\right) \in J$ se verifica:

$$
{ }^{a} \Delta^{b} f(x)=\left.\left.\Delta_{p 1} \Delta_{p 2} \ldots \Delta_{p n} f(x)\right|_{a_{p n}} ^{b_{p n}} \ldots||_{a_{p 2}}^{b_{p 2}}\right|_{a_{p 1}} ^{b_{p 1}}
$$


Prueba. (Por inducción sobre $n$ ). Para $n=1$ se tiene: ${ }^{a} \Delta^{b} f(x)=f(b)-f(a)=$ $\left.\Delta f(x)\right|_{a} ^{b}=\left.\Delta_{1} f(x)\right|_{a} ^{b}$.

Asumiendo la hipótesis de inducción para $n$, sea $p$ una permutación de $\{1, \ldots, n, n+1\}$.

Sean $a=\left(a_{1}, \ldots, a_{n+1}\right), b=\left(b_{1}, \ldots, b_{n+1}\right)$ y denotemos $a^{\prime}, b^{\prime}$ los puntos de $\mathbb{R}^{n}$ que resultan de extraer la componente de lugar $p(n+1)$ en $a$ y $b$ respectivamente. Sea $f\left(x_{1}, \ldots, x_{n+1}\right)$ una función de $n+1$ variables. Consideremos las siguientes funciones de $n$ variables:

$$
\begin{aligned}
& g\left(x_{1}, \ldots, x_{p(n+1)-1}, x_{p(n+1)+1}, \ldots, x_{n+1}\right)=f\left(x_{1}, \ldots, x_{p(n+1)-1}, a_{p(n+1)}, x_{p(n+1)+1}, \ldots, x_{n+1}\right) \\
& h\left(x_{1}, \ldots, x_{p(n+1)-1}, x_{p(n+1)+1}, \ldots, x_{n+1}\right)=f\left(x_{1}, \ldots, x_{p(n+1)-1}, b_{p(n+1)}, x_{p(n+1)+1}, \ldots, x_{n+1}\right)
\end{aligned}
$$

Se tiene:

$$
h-g=\left.\Delta_{p(n+1)} f\right|_{a_{p(n+1)}} ^{b_{p(n+1)}} .
$$

Ahora definamos los siguientes conjuntos:

$$
\begin{aligned}
& A=\left\{K \in\{0,1\}^{n+1} \mid K_{p(n+1)}=1\right\} \\
& B=\left\{K \in\{0,1\}^{n+1} \mid K_{p(n+1)}=0\right\}
\end{aligned}
$$

Se tiene:

$$
\begin{aligned}
\Delta^{b} f(x) & =\sum_{K \in A}(-1)^{|K|} f((a \vee b)(K))+\sum_{K \in B}(-1)^{|K|} f((a \vee b)(K)) \\
a^{\prime} \Delta^{b^{\prime}} h(x) & =\sum_{K \in\{0,1\}^{n}}(-1)^{|K|} h\left(\left(a^{\prime} \bigvee b^{\prime}\right)(K)\right)=\sum_{K \in B}(-1)^{|K|} f((a \vee b)(K)) \\
a^{\prime} \Delta^{b^{\prime}} g(x) & =\sum_{K \in\{0,1\}^{n}}(-1)^{|K|} g\left(\left(a^{\prime} \bigvee b^{\prime}\right)(K)\right)=-\sum_{K \in A}(-1)^{|K|} f((a \vee b)(K)),
\end{aligned}
$$

por lo que

$$
{ }^{a} \Delta^{b} f(x)={ }^{a^{\prime}} \Delta^{b^{\prime}}(h(x)-g(x))={ }^{a^{\prime}} \Delta^{b^{\prime}}\left(\Delta_{p(n+1)} f(x) \mid \begin{array}{l}
b_{p(n+1)} \\
a_{p(n+1)}
\end{array}\right),
$$

y usando la hipótesis de inducción:

$$
{ }^{a} \Delta^{b} f(x)=\left.\left.\Delta_{p 1} \Delta_{p 2} \ldots \Delta_{p n}\left(\left.\Delta_{p(n+1)} f(x)\right|_{a_{p(n+1)}} ^{b_{p(n+1)}}\right)\right|_{a_{p n}} ^{b_{p n}} \ldots||_{a_{p 2}}^{b_{p 2}}\right|_{a_{p 1}} ^{b_{p 1}}
$$

lo que completa la prueba por inducción.

Teorema 2. (Incremento multivariado del producto de funciones univariadas). Sean $f_{i}: J_{i} \longrightarrow \mathbb{R}, i=1, \ldots, n$, funciones reales de una variable real, respectivamente definidas en intervalos $J_{i} \subseteq \mathbb{R}$. Sea $J=J_{1} \times \cdots \times J_{n}$ y considérese la función $f: J \longrightarrow \mathbb{R}$ definida por

$$
f\left(x_{1}, x_{2}, \ldots, x_{n}\right)=f_{1}\left(x_{1}\right) \cdot f_{2}\left(x_{2}\right) \cdot \ldots \cdot f_{n}\left(x_{n}\right) .
$$

Entonces, dados $a=\left(a_{1}, \ldots, a_{n}\right), b=\left(b_{1}, \ldots, b_{n}\right) \in J$, se cumple

$$
{ }^{a} \Delta^{b} f(x)=\left.\left.\left.\Delta f_{1}\left(x_{1}\right)\right|_{a_{1}} ^{b_{1}} \cdot \Delta f_{2}\left(x_{2}\right)\right|_{a_{2}} ^{b_{2}} \cdot \ldots \cdot \Delta f_{n}\left(x_{n}\right)\right|_{a_{n}} ^{b_{n}}
$$


En particular:

$$
{ }^{a} \Delta^{b} x={ }^{a} \Delta^{b} x_{1} x_{2} \cdots x_{n}=\left(b_{1}-a_{1}\right) \cdot\left(b_{2}-a_{2}\right) \cdot \ldots \cdot\left(b_{n}-a_{n}\right) .
$$

Prueba. Consecuencia inmediata del teorema 1 y del hecho que para todo $i=1, \ldots, n$ se tiene:

$$
\begin{aligned}
\left.\Delta_{i} f_{1}\left(x_{1}\right) \cdot f_{2}\left(x_{2}\right) \cdot \ldots \cdot f_{i}\left(x_{i}\right) \cdot c_{i}\right|_{a_{i}} ^{b_{i}}= & f_{1}\left(x_{1}\right) \cdot f_{2}\left(x_{2}\right) \cdot \ldots \cdot f_{i}\left(b_{i}\right) \cdot c_{i} \\
& \quad-f_{1}\left(x_{1}\right) \cdot f_{2}\left(x_{2}\right) \cdot \ldots \cdot f_{i}\left(a_{i}\right) \cdot c_{i} \\
= & f_{1}\left(x_{1}\right) \cdot f_{2}\left(x_{2}\right) \cdot \ldots \cdot f_{i-1}\left(x_{i-1}\right) \cdot\left(f_{i}\left(b_{i}\right)-f_{i}\left(a_{i}\right)\right) \cdot c_{i} \\
= & \left.f_{1}\left(x_{1}\right) \cdot f_{2}\left(x_{2}\right) \cdot \ldots \cdot f_{i-1}\left(x_{i-1}\right) \cdot \Delta f_{i} \cdot c_{i}\right|_{a_{i}} ^{b_{i}},
\end{aligned}
$$

donde $c_{n}=1$ y para $1 \leq i<n, c_{i}$ es la constante $\left.\left.\Delta f_{i+1}\left(x_{i+1}\right)\right|_{a_{i+1}} ^{b_{i+1}} \cdot \ldots \cdot \Delta f_{n}\left(x_{n}\right)\right|_{a_{n}} ^{b_{n}}$.

Teorema 3. (Aditividad). Sea $J \subseteq \mathbb{R}^{n}$ una $n$-celda, sea $\left\{J_{1}, \ldots, J_{m}\right\}$ una partición de $J$ en $m$ subceldas. Sea $f$ una función real definida en $J$. Entonces

$$
\Delta_{J} f=\sum_{i=1}^{m} \Delta_{J_{i}} f
$$

Prueba. Sea $J=\left[a_{1}, b_{1}\right] \times \ldots \times\left[a_{n}, b_{n}\right]$ con $a_{i} \leq b_{i}$ para todo $i=1, \ldots, n$. Basta probar el teorema para el caso $m=2$, es decir cuando $J$ se particiona en dos subceldas yuxtapuestas $J_{1}$ y $J_{2}$. Por el teorema 1 , podemos suponer que $J_{1}$ y $J_{2}$ están yuxtapuestas paralelamente al eje $x_{1}$, por lo que deben ser de la forma

$$
J_{1}=\left[a_{1}, c\right] \times\left[a_{2}, b_{2}\right] \times \ldots \times\left[a_{n}, b_{n}\right], \quad J_{2}=\left[c, b_{1}\right] \times\left[a_{2}, b_{2}\right] \times \ldots \times\left[a_{n}, b_{n}\right]
$$

para algún $c \in\left[a_{1}, b_{1}\right]$.

Se tiene:

$$
\Delta_{J} f={ }^{a} \Delta^{b} f(x), \quad \Delta_{J_{1}} f={ }^{a} \Delta^{b^{\prime}} f(x), \quad \text { y } \quad \Delta_{J_{2}} f={ }^{a^{\prime}} \Delta^{b} f(x),
$$

donde

$$
\begin{array}{ll}
a=\left(a_{1}, a_{2}, \ldots, a_{n}\right) & b=\left(b_{1}, b_{2}, \ldots, b_{n}\right) \\
a^{\prime}=\left(c, a_{2}, \ldots, a_{n}\right) & b^{\prime}=\left(c, b_{2}, \ldots, b_{n}\right)
\end{array}
$$

Definamos: $\mathcal{A}=\left\{K \in\{0,1\}^{n} \mid K_{1}=1\right\}, \quad \mathcal{B}=\left\{K \in\{0,1\}^{n} \mid K_{1}=0\right\}$.

Nótese que:

$$
\begin{aligned}
\left(\left(a \vee b^{\prime}\right)(K)\right)_{1} & =a_{1} & & \text { si } K \in \mathcal{A}) \\
\left(\left(a \vee b^{\prime}\right)(K)\right)_{1} & =c & & \text { si } K \in \mathcal{B} \\
\left(\left(a^{\prime} \vee b\right)(K)\right)_{1} & =c & & \text { si } K \in \mathcal{A} \\
\left(\left(a^{\prime} \vee b\right)(K)\right)_{1} & =b_{1} & & \text { si } K \in \mathcal{B}
\end{aligned}
$$


Por brevedad escribamos:

$$
\begin{aligned}
P=\sum_{K \in \mathcal{A}}(-1)^{|K|} f((a \vee b)(K)), & Q=\sum_{K \in \mathcal{B}}(-1)^{|K|} f((a \vee b)(K)), \\
R=\sum_{K \in \mathcal{B}}(-1)^{|K|} f\left(\left(a \vee b^{\prime}\right)(K)\right), & S=\sum_{K \in \mathcal{A}}(-1)^{|K|} f\left(\left(a^{\prime} \vee b\right)(K)\right),
\end{aligned}
$$

y tomemos nota de la siguiente igualdad:

$$
P+Q={ }^{a} \Delta^{b} f
$$

Ahora, por (2) tenemos: $P=\sum_{K \in \mathcal{A}}(-1)^{|K|} f\left(\left(a \vee b^{\prime}\right)(K)\right)$; luego:

$$
P+R={ }^{a} \Delta^{b^{\prime}} f
$$

Además, por (5) tenemos: $Q=\sum_{K \in \mathcal{B}}(-1)^{|K|} f\left(\left(a^{\prime} \vee b\right)(K)\right)$; luego:

$$
Q+S={ }^{a^{\prime}} \Delta^{b} f
$$

A continuación, consideremos la biyección $\phi: \mathcal{A} \longrightarrow \mathcal{B}$ definida por $(\phi(K))_{j}=K_{j}$ para todo $1<j \leq n$. Se tiene:

$$
(-1)^{|K|}=-(-1)^{|\phi(K)|} \quad \text { para todo } \quad K \in \mathcal{A} .
$$

Luego, por (3) y (4) obtenemos: $S=-R$, y, por (8):

$$
Q-R={ }^{a^{\prime}} \Delta^{b} f
$$

Los resultados (1), (6), (7) y (9) nos permiten escribir:

$$
\begin{aligned}
\Delta_{J} f & ={ }^{a} \Delta^{b} f \\
& =P+Q \\
& =P+R-R+Q \\
& =(P+R)+(S+Q) \\
& ={ }^{a} \Delta^{b^{\prime}} f+{ }^{a^{\prime}} \Delta^{b} f \\
& =\Delta_{J_{1}} f+\Delta_{J_{2}} f
\end{aligned}
$$

Teorema 4. (Caracterización de funciones con incremento multivariado nulo en las subceldas de $J$ ). Sea $J \subseteq \mathbb{R}^{n}$ una $n$-celda. Sea $f: J \longrightarrow \mathbb{R}$. Una condición necesaria y suficiente para que $\Delta_{K} f=0$ para toda $n$-subcelda $K \subseteq J$, es que $f$ sea una suma de $n$ funciones $f_{i}, i=1, \ldots, n$, que respectivamente no dependen de la variable $x_{i}$.

Prueba.

Suficiencia: Como $f_{i}$ no depende de $x_{i}$, entonces $\left.\Delta_{i} f_{i}(x)\right|_{a_{i}} ^{b_{i}}=0$ para todo $i=1, \ldots, n$ y el teorema 1 nos permite escribir $\Delta_{K} f_{i}=0$ para todo $i=1, \ldots, n$. Por linealidad de $\Delta_{K}$, obtenemos: $\Delta_{K} f=0$ para toda $n$-celda $K \subseteq J$. 
Necesidad: (Por inducción sobre $n$ ). Para $n=1$, si $\left.\Delta f(x)\right|_{a} ^{t}=0$ para todos $a, t \in J \subseteq \mathbb{R}$, entonces $f(t)=f(a)$ para todo $t \in J$, es decir $f$ no depende de $t$.

Asumiendo la hipótesis de inducción para $n$, sea $J=\left[a_{1}, b_{1}\right] \times \cdots \times\left[a_{n}, b_{n}\right] \times[a, b] \subseteq$ $\mathbb{R}^{n+1}$ una $(n+1)$-celda y sea $f: J \longrightarrow \mathbb{R}$ tal que $\Delta_{K} f=0$ para toda $(n+1)$-subcelda $K \subseteq J$. Para cada $t \in[a, b]$ consideremos la función $g_{t}$ definida en $\left[a_{1}, b_{1}\right] \times \cdots \times\left[a_{n}, b_{n}\right] \subseteq$ $\mathbb{R}^{n}$ mediante:

$$
g_{t}\left(t_{1}, \ldots, t_{n}\right)=f\left(t_{1}, \ldots, t_{n}, t\right)-f\left(t_{1}, \ldots, t_{n}, a_{n+1}\right) .
$$

Para cada $n$-subcelda $K$ de la $n$-celda $\left[a_{1}, b_{1}\right] \times \cdots \times\left[a_{n}, b_{n}\right]$, por teorema 1 se tiene:

$$
\Delta_{K} g_{t}=\Delta_{K \times[a, b]} f=0 ;
$$

entonces, por hipótesis de inducción concluimos que $g_{t}\left(t_{1}, \ldots, t_{n}\right)$ es una suma de $n$ funciones $\left(g_{t}\right)_{i}\left(t_{1}, \ldots, t_{n}\right)(i=1, \ldots, n)$ que respectivamente no dependen de la respectiva variable $t_{i}$. Esto, junto con el hecho que la función $f\left(t_{1}, \ldots, t_{n}, a_{n+1}\right)$ no depende de $t_{n+1}$, muestra que $f\left(t_{1}, \ldots, t_{n}, t_{n+1}\right)$ es una suma de $(n+1)$ funciones $f_{i}\left(t_{1}, \ldots, t_{n}, t_{n+1}\right)$ $(i=2, \ldots, n+1)$ que respectivamente no dependen de la correspondiente variable $t_{i}$.

\section{La noción de $n$-continuidad}

Mediante el incremento multivariado podemos definir una noción de " $n$-continuidad" que guarda la misma relación existente entre las nociones habituales de incremento y continuidad.

Definición D. Sea $J \subseteq \mathbb{R}^{n}$ una $n$-celda. Sea $a \in J$ y sea $f: J \longrightarrow \mathbb{R}$. Diremos que $f$ es $n$-continua en a si se verifica la siguiente condición: para todo $\epsilon>0$ existe $\delta>0$ tal que para todo $y \in J$ :

$$
\|y-a\|<\delta \Longrightarrow\left|{ }^{a} \Delta^{y} f\right|<\epsilon .
$$

Diremos que $f$ es $n$-continua si $f$ es $n$-continua en $x$, para todo $x \in J$.

La relación entre las nociones de continuidad y $n$-continuidad queda establecida en nuestro'ultimo teorema:

Teorema 5. Caracterización de continuidad). Sea $J \subseteq \mathbb{R}^{n}$ una $n$-celda. Sea $a \in J$ y sea $f: J \longrightarrow \mathbb{R}$. Una condición necesaria y suficiente para que $f$ sea continua en $a$ es que $f$ sea separadamente continua en $a$ con respecto a cualquier subconjunto propio del conjunto de las $n$ componentes $x_{i}$ de la variable $n$-dimensional $x=\left(x_{1}, \ldots, x_{n}\right)$ y que, además, $f$ sea $n$-continua en $a$.

Prueba. Supongamos en primer lugar que $f$ es continua en $a \in J$. Es claro que entonces $f$ es separadamente continua en $a$ con respecto a cualquier subconjunto propio del conjunto de las $n$ componentes $x_{i}$ de la variable $n$-dimensional $x=\left(x_{1}, \ldots, x_{n}\right)$. Probaremos que $f$ es $n$-continua en $a$.

Sea $\epsilon>0$. Entonces existe $\delta>0$ tal que para todo $y \in J$ :

$$
\|y-a\|<\delta \Longrightarrow|f(y)-f(a)|<\frac{\epsilon}{2^{n}}
$$


Observemos que para todo $y \in J$ y todo $K \in\{0,1\}^{n}$, se verifica:

$$
\|y-a\|<\delta \Longrightarrow\|(a \vee y)(K)-a\|<\delta,
$$

por lo tanto, para todo $y \in J$ y todo $K \in\{0,1\}^{n}$ :

$$
\|y-a\|<\delta \Longrightarrow|f((a \vee y)(K))-f(a)|<\frac{\epsilon}{2^{n}} .
$$

Luego:

$$
\|y-a\|<\delta \Longrightarrow \sum_{K \in\{0,1\}^{n}}|f((a \vee y)(K))-f(a)|<\sum_{K \in\{0,1\}^{n}} \frac{\epsilon}{2^{n}}=\epsilon,
$$

ya que card $\{0,1\}^{n}=2^{n}$. Ahora, si denotamos

$$
A=\left\{K \in\{0,1\}^{n}|| K \mid \text { es par }\right\}, \quad B=\left\{K \in\{0,1\}^{n}|| K \mid \text { es impar }\right\}
$$

se tiene:

$$
{ }^{a} \Delta^{y} f(x)=\sum_{K \in A} f((a \vee y)(K))-\sum_{K \in B} f((a \vee y)(K))
$$

y como card $A=$ card $B=2^{n-1}$, entonces al sumar y restar $2^{n-1}$ veces en el lado derecho la cantidad $f(a)$, podemos escribir:

$$
{ }^{a} \Delta^{y} f(x)=\sum_{K \in A}(f((a \vee y)(K))-f(a))-\sum_{K \in B}(f((a \vee y)(K))-f(a))
$$

y, usando (10):

$$
\begin{aligned}
\left|{ }^{a} \Delta^{y} f(x)\right| & \leq\left|\sum_{K \in A}(f((a \vee y)(K))-f(a))\right|+\left|\sum_{K \in B}(f((a \vee y)(K))-f(a))\right| \\
& \leq \sum_{K \in A}|(f((a \vee y)(K))-f(a))|+\sum_{K \in B}|(f((a \vee y)(K))-f(a))| \\
& =\sum_{K \in\{0,1\}^{n}}|(f((a \vee y)(K))-f(a))|<\epsilon,
\end{aligned}
$$

siempre que $y \in J$ y $\|y-a\|<\delta$. Esto muestra que $f$ es $n$-continua en $a$.

Veamos ahora la otra parte de la prueba. Sea $\epsilon>0$. Asumiendo las hipótesis, existe $\delta>0$ tal que

$$
\left|{ }^{a} \Delta^{y} f(x)\right|<\frac{\epsilon}{2^{n}}
$$

además, para cada una de las $2^{n}-1 K \in\{0,1\}^{n}$ distintas de $(0, \ldots, 0)$, se tiene:

$$
|f((a \vee y)(K))-f(a)|<\frac{\epsilon}{2^{n}} .
$$


Recordando ahora la igualdad (11) se tiene:

$$
(f(y))-f(a))={ }^{a} \Delta^{y} f-\sum_{K \in A^{\prime}}(f((a \vee y)(K))-f(a))+\sum_{K \in B}(f((a \vee y)(K))-f(a)),
$$

donde

$$
A^{\prime}=\left\{K \in\{0,1\}^{n}|| K \mid \text { es par no nulo }\right\}, \quad B=\left\{K \in\{0,1\}^{n}|| K \mid \text { es impar }\right\}
$$

Entonces podemos escribir:

$$
\begin{aligned}
\mid f(y))-f(a) \mid & \leq\left|{ }^{a} \Delta^{y} f(x)\right|+\sum_{K \in A^{\prime}}|f((a \vee y)(K))-f(a)|+\sum_{K \in B}|f((a \vee y)(K))-f(a)| \\
& <\frac{\epsilon}{2^{n}}+\left(2^{n}-1\right) \frac{\epsilon}{2^{n}}=\epsilon
\end{aligned}
$$

\section{Avance de algunas aplicaciones}

En [1] hicimos una reelaboración de la integral de Riemann para funciones reales de una variable real. El incremento multivariado permite generalizar dicho trabajo para funciones reales multivariadas, para lo cual en una futura publicación comenzaremos por generalizar la noción de pre-primitiva que allí dimos. Con esa noción probaremos que la función multivariada $f$, acotada en la $n$-celda $J$, es Riemann-integrable en las subceldas compactas de $J$, si y sólo si para cualquiera subcelda $K \subseteq J$ y para cualesquier par de pre-primitivas $F$ y $G$ de $f$ en $J$ (siempre existen), se verifica:

$$
\Delta_{K} F=\Delta_{K} G
$$

y, en tal caso:

$$
\int_{K} f(x) d x=\Delta_{K} F(x)
$$

donde $F$ es cualquier pre-primitiva de $f$ en $K$, que es la versión isomorfa del segundo Teorema Fundamental del Cálculo.

Otra noción que fluye de manera natural de la noción de incremento multivariado, es la de derivada multivariada de una función multivariada $f$, por medio de la cual tendremos la siguiente versión isomorfa del primer Teorema Fundamental del Cálculo:

$$
\int_{J} f^{\prime}(x) d x=\Delta_{J} f
$$

donde se supone que $f$ es una función multivariada Riemann-integrable en la $n$-celda $J$, que posee derivada multivariada $f^{\prime}(x)$ en cada punto $x \in J$. 


\section{Referencias}

[1] Alarcón, W. (1992) "Integral y primitivas de Riemann", Ciencias Matemáticas, 2(2): $41-47$.

[2] Bartle, R. (1976) The Elements of Real Analysis. 2a. Edición, John Wiley \& Sons, New York.

[3] Lang, S. (1983) Real Analysis. 2a. Edición, Addison-Wesley, Reading, Mass.

[4] Spivak, M. Calculus on Manifolds. 